RKCL5508

\title{
RELAXATION OF CONCENTRATION PERTURBATION IN CHEMICAL KINETIC SYSTEMS
}

\author{
Tibor Nagy and Tamás Turányi* \\ Institute of Chemistry, Eötvös University (ELTE) \\ H-1518 Budapest, P.O. Box 32, Hungary \\ Received January 16, 2009, in revised form January 23, 2009, accepted January 26, 2009
}

\begin{abstract}
In a linear approximation, the relaxation of a concentration perturbation can be described by a matrix exponential, which can be evaluated using Jordan decomposition. In time-scale analysis, this approach has advantages when the Jacobian has degenerate eigenvalues, which may occur when the mechanism contains identical rate constants, characteristic to tropospheric chemistry and lowtemperature combustion.
\end{abstract}

Keywords: Time scales, mechanism reduction, manifold, CSP, ILDM

\section{INTRODUCTION}

Concentration perturbation means that the concentrations of one or several species are altered in a much shorter time than the characteristic time scale of the system. Calculation of the effect of concentration perturbation is straightforward in computer modelling using detailed reaction mechanisms [1], [2]. In laboratory experiments, such a concentration perturbation can be achieved for example by adding a precursor of one (or several) of the species and applying laser flash photolysis.

The response of chemical kinetic models to the simultaneous perturbations in the values of several concentrations was investigated by Lam and Goussis [3] and Maas and Pope [4]. They developed a series of Computational Singular Perturbation (CSP) and Intrinsic Low Dimensional Manifold (ILDM) methods, respectively, for the efficient reduction of large reaction mechanisms using time-scale analysis. The background of these methods is that a perturbation in

* Corresponding author. E-mail: turanyi@chem.elte.hu 
the concentration space can be decomposed according to the eigenvectors of the Jacobian of the kinetic system of differential equations, and their time evolutions depend on the corresponding eigenvalues. Lu and Law [5] developed a complex CSP method, which can also handle the oscillating relaxation in the case of complex eigenvalues.

It is usually assumed that the Jacobian is diagonalizable, that is it has a complete system of linearly independent eigenvectors. However, if some of the eigenvalues of the Jacobian are degenerate, then a more general method is required to describe the time evolution of a concentration perturbation.

\section{THEORY}

\section{Time evolution of a concentration perturbation}

Let a chemical kinetics model be described by the following initial value problem

$$
\frac{\mathrm{d} \mathbf{c}}{\mathrm{d} t}=\mathbf{f}(\mathbf{c}, \mathbf{p}) \quad \mathbf{c}(0)=\mathbf{c}_{0},
$$

where $t$ is time, $\mathbf{c}$ is the $n$-vector of variables, $\mathbf{p}$ is the vector of parameters, $\mathbf{c}_{0}$ is the vector of the initial values of the variables, and $\mathbf{f}(\mathbf{c}, \mathbf{p})$ is the righthand-side of the kinetic differential equation system. If initially a small concentration perturbation $\Delta \mathbf{c}_{0}$ is applied to the system, the production rates of the species can be estimated by local linearization of $\mathbf{f}$ :

$\frac{\mathrm{d} \mathbf{c}}{\mathrm{d} t}+\frac{\mathrm{d} \Delta \mathbf{c}}{\mathrm{d} t}=\frac{\mathrm{d}(\mathbf{c}+\Delta \mathbf{c})}{\mathrm{d} t}=\mathbf{f}(\mathbf{c}+\Delta \mathbf{c}, \mathbf{p})=\mathbf{f}(\mathbf{c}, \mathbf{p})+\mathbf{J} \Delta \mathbf{c}+O\left(\Delta \mathbf{c}^{2}\right)$,

where $\mathbf{J}=\mathrm{d} \mathbf{f} / \mathrm{d} \mathbf{c}$ is the Jacobian of the system of ordinary differential equations. The Jacobian is a real non-symmetric $n \times n$ matrix. From equations (1) and (2), it follows (see e.g. [6]) that the time evolution of a small concentration perturbation $\Delta \mathbf{c}$ can be described by the variational equation (also called sensitivity equation):

$$
\frac{\mathrm{d} \Delta \mathbf{c}}{\mathrm{d} t}=\mathbf{J} \Delta \mathbf{c} \quad \Delta \mathbf{c}(0)=\Delta \mathbf{c}_{0} .
$$


Assuming that the Jacobian is constant $\mathbf{J}_{0}$ in a short time interval, the solution can be approximated by multiplying $\Delta \mathbf{c}_{0}$ with a matrix exponential, called the time-propagator matrix.

$\Delta \mathbf{c}=e^{\mathbf{J}_{0} t} \Delta \mathbf{c}_{0}$.

\section{Decomposition of the time-propagator matrix into eigenmodes}

In order to evaluate the matrix exponential of $\mathbf{J}_{0} t$, it is practical to decompose $\mathbf{J}_{0}$ into a Jordan canonical form $\boldsymbol{J}$ with an appropriate invertible matrix $\mathbf{P}$ because $\mathbf{J}_{0}=\mathbf{P} \boldsymbol{J} \mathbf{P}^{-1}$ implies $e^{\mathbf{J}_{0} t}=\mathbf{P} e^{J t} \mathbf{P}^{-1}$. The eigenvalue equation of matrix $\mathbf{J}_{0}$, where $\boldsymbol{\Lambda}$ denotes the diagonal matrix of the eigenvalues, and matrix $\mathbf{X}$ contains the right eigenvectors as columns vectors.

$$
\mathbf{J}_{0} \mathbf{X}=\mathbf{X} \boldsymbol{\Lambda} \quad \boldsymbol{\Lambda}=\operatorname{diag}\left(\lambda_{1}, \ldots, \lambda_{n}\right) \quad \mathbf{X}=\left[\begin{array}{lll}
\mathbf{x}_{1} & \ldots & \mathbf{x}_{n}
\end{array}\right]
$$

A multiple eigenvalue can be characterized by its algebraic and geometric multiplicities. The algebraic multiplicity $a(\lambda)$ of eigenvalue $\lambda$ is the multiplicity of root $\lambda$ of the characteristic polynomial. The geometric multiplicity $g(\lambda)$ is the dimension of the eigenspace of $\lambda$, which is the number of linearly independent eigenvectors belonging to $\lambda$. If $g(\lambda)<a(\lambda)$ for a multiple eigenvalue, then we call it a degenerate eigenvalue. If at least one of the eigenvalues of $\mathbf{J}_{0}$ is degenerate, then the eigenvectors do not form a complete system, thus $\mathbf{J}_{0}$ cannot be diagonalized.

For the general case, the Jordan decomposition of the Jacobian should be considered to evaluate the matrix exponential. The Jordan basis consists of generalized right eigenvectors $\left\{\mathbf{x}_{i j k}\right\}$, which are defined by the generalization of the eigenvalue equation.

$$
\begin{array}{lrr}
\left(\mathbf{J}_{0}-\lambda_{i} \mathbf{I}\right)^{k} \mathbf{x}_{i j k}=\mathbf{0} & i=1, \ldots, m & j=1, \ldots, m_{i} \quad k=1, \ldots, m_{i j} \\
n=\sum_{i=1}^{m} a\left(\lambda_{i}\right) & m_{i}=g\left(\lambda_{i}\right) \quad a\left(\lambda_{i}\right)=\sum_{j=1}^{m_{i}} m_{i j}
\end{array}
$$

There are $m$ different $\lambda_{i}$ eigenvalues of $\mathbf{J}_{0}$ and $\mathbf{x}_{i j k}$ denotes the corresponding generalized right eigenvectors. The "ordinary" eigenvectors are the generalized 
eigenvectors with $k=1$. Each linearly independent $\mathbf{x}_{i j 1}$ eigenvector initiates a Jordan chain, which contains further $m_{i j}-1$ generalized eigenvectors that are defined by equation (7). Here $j$ is the index of the Jordan chain of an eigenvalue and $k$ is the index of the generalized eigenvector within a Jordan chain.

$$
\left(\mathbf{J}_{0}-\lambda_{i} \mathbf{I}\right) \mathbf{x}_{i j k}=\mathbf{x}_{i j k-1} \quad k=2, \ldots, m_{i j}
$$

Equation (7) also fixes the relative lengths and phases of generalized eigenvectors within a chain. Matrix $\mathbf{J}_{0}$ can be decomposed into a Jordan canonical form, with matrices $\mathbf{P}=\left[\ldots, \mathbf{x}_{i j k}, \ldots\right]$ and $\mathbf{P}^{-1}=\left[\ldots, \mathbf{y}_{i j k}, \ldots\right]^{+}=\left[\ldots, \overline{\mathbf{y}}_{i j k}, \ldots\right]^{T}$. Here, $\left\{\mathbf{y}_{i j k}\right\}$ are the scaled generalized left eigenvectors, which are in reciprocal relation with the right eigenvectors regarding the Hermitian scalar product: $\mathbf{y}_{i j k}^{+} \mathbf{x}_{I J K}=\delta_{i I} \delta_{j J} \delta_{k K}$. The Jordan form of a matrix is built up of Jordan blocks $\boldsymbol{J}_{i j}$ of size $m_{i j} \times m_{i j}$. Each block belongs to a Jordan chain having the same indices $i j$.

$$
\mathbf{J}_{0}=\mathbf{P} \boldsymbol{J} \mathbf{P}^{-1} \quad \boldsymbol{J}=\operatorname{blockdiag}\left(\ldots, \boldsymbol{J}_{i j}, \ldots\right)
$$

A Jordan block $\boldsymbol{J}_{q}$ is of the form $\boldsymbol{\Lambda}_{q}+\mathbf{N}_{q}$ of size $m_{i j} \times m_{i j}$, where $q=i j$ and

$$
\boldsymbol{\Lambda}_{q}=\lambda_{i} \mathbf{I}, \quad \mathbf{N}_{q}=\left[\begin{array}{cccc}
0 & 1 & 0 & \cdots \\
0 & 0 & 1 & \ddots \\
\vdots & \ddots & \ddots & \ddots
\end{array}\right]
$$

For any matrix $\mathbf{J}_{0}$ there exists a Jordan basis and the Jordan form is uniquely determined up to a permutation of its Jordan blocks ([7], point 12.2). Exponential of a block-diagonal matrix can be calculated by blocks.

$$
e^{\mathbf{J}_{0} t}=e^{\mathbf{P J} \mathbf{P}^{-1} t}=\mathbf{P} e^{J t} \mathbf{P}^{-1}=\mathbf{P} \cdot \operatorname{blockdiag}\left(\ldots, \exp \left(\boldsymbol{J}_{i j} t\right), \ldots\right) \cdot \mathbf{P}^{-1}
$$

The power of a block can be calculated using Newton's binomial formula ([7], point 12.3).

$$
\left(\boldsymbol{\Lambda}_{q}+\mathbf{N}_{q}\right)^{r}=\sum_{l=0}^{r}\left(\begin{array}{l}
r \\
l
\end{array}\right) \lambda_{q}^{l} \mathbf{N}_{q}^{r-l} \quad r=m_{i j}
$$


If $\mathbf{N}_{q}$ is an $r \times r$ matrix, then $\mathbf{N}_{q}{ }^{r}=\mathbf{0}$. Therefore, the exponential of a Jordan block of size $r \times r$ can be calculated as ([7], point 46.1):

$$
e^{J_{q} t}=e^{\Lambda_{q} t} e^{\mathbf{N}_{q} t}=e^{\lambda_{q} t} \sum_{l=1}^{r-1} \frac{t^{l}}{l !} \mathbf{N}_{q}^{l}=e^{\lambda_{q} t}\left[\begin{array}{ccccc}
1 & t & t^{2} / 2 ! & \cdots & t^{r-1} /(r-1) ! \\
0 & 1 & t & t^{2} / 2 ! & \cdots \\
\vdots & \ddots & \ddots & \ddots & \vdots \\
0 & \cdots & 0 & 1 & t \\
0 & \cdots & \cdots & 0 & 1
\end{array}\right]
$$

Due to the block-diagonal structure of $e^{J t}$, only products of vectors $\mathbf{x}_{i j k}$ and $\mathbf{y}_{i j l}^{+}$from the same $i j$ Jordan chain will remain when equation (10) is evaluated.

$e^{\mathbf{J}_{0} t}=\sum_{i=1}^{m} e^{\lambda_{i} t} \sum_{j=1}^{m_{i}} \sum_{k=1}^{m_{i j}} \sum_{l=k}^{m_{i j}} \frac{t^{l-k}}{(l-k) !} \mathbf{x}_{i j k} \mathbf{y}_{i j l}^{+}=\sum_{i=1}^{m} e^{\lambda_{i} t} \sum_{j=1}^{m_{i}}\left(\sum_{k=1}^{m_{i j}} \mathbf{P}_{i j k}+\sum_{k=1}^{m_{i j}-1} \sum_{l=k+1}^{m_{i j}} \frac{t^{l-k}}{(l-k) !} \mathbf{F}_{i j l \rightarrow k}\right)$

Matrix exponential $e^{\mathbf{J}_{0} t}$ transforms the initial perturbation $\Delta \mathbf{c}_{0}$ into modes parallel with $\mathbf{x}_{i j k}$ by operators of $\mathbf{F}_{i j l \rightarrow k}=\mathbf{x}_{i j k} \mathbf{y}_{i j l}^{+}$. Each mode evolves in time according to $e^{\lambda_{i} t} t^{l-k} /(l-k)$ !. For modes $k=l$, the change is purely exponential and operators $\mathbf{F}_{i j k \rightarrow k}$ simplify to skew-projectors $\mathbf{P}_{i j k}=\mathbf{x}_{i j k} \mathbf{y}_{i j k}^{+}$, which are idempotent and disjoint, that is $\mathbf{P}_{i j k} \mathbf{P}_{I J K}=\delta_{i I} \delta_{j J} \delta_{k K} \mathbf{P}_{i j k}$.

\section{The case of a diagonalizable Jacobian}

If the Jacobian is diagonalizable, then the Jordan decomposition coincides with the eigenvalue-eigenvector decomposition $(\mathbf{P}=\mathbf{X}, \boldsymbol{J}=\boldsymbol{\Lambda})$ and $m_{i j}=1$ for all Jordan chains, thus only projector type of operations will remain and the modes will not have $t$ power factor.

$$
\Delta \mathbf{c}=e^{\mathbf{J}_{\mathbf{0}} t} \Delta \mathbf{c}_{0}=\sum_{\mathbf{i}=1}^{\mathbf{m}} e^{\lambda_{\mathbf{i}} t} \sum_{\mathbf{j}=1 \mathbf{k}=1}^{\mathbf{m}_{\mathbf{i}}} \sum_{\mathbf{i}=1}^{\mathbf{m}_{\mathbf{j}}} \mathbf{P}_{\mathbf{i j k}} \Delta \mathbf{c}_{0}=\sum_{\mathbf{l}=1}^{\mathbf{n}} e^{\lambda_{\mathbf{l}} t} \mathbf{P}_{\mathbf{l}} \Delta \mathbf{c}_{0}
$$

In this case the eigenvalues may be either real or complex. 


\section{Interpretation of the real modes in the general case}

The generalized eigenvectors belonging to a real eigenvalue can always be selected to be real vectors ([7], point 12.1). The operation described by matrix $\mathbf{F}_{i j l \rightarrow k}$ can be decomposed into three operations: a skew-projection onto the space spanned by $\mathbf{x}_{i j l}$ along the directions of the basis vectors different from $\mathbf{x}_{i j l}$, a rotation from direction $\mathbf{x}_{i j l}$ to direction $\mathbf{x}_{i j k}$, and finally a rescaling by $x_{i j k} / x_{i j l}$.

$\mathbf{x}_{i j k} \mathbf{y}_{i j l}^{T}=\mathbf{x}_{i j k}\left(\hat{\mathbf{x}}_{i j l}^{T} \hat{\mathbf{x}}_{i j l}\right) \mathbf{y}_{i j l}^{T}=\left(x_{i j k} / x_{i j l}\right) \cdot\left(\hat{\mathbf{x}}_{i j k} \hat{\mathbf{x}}_{i j l}^{T}\right) \cdot\left(\mathbf{x}_{i j l} \mathbf{y}_{i j l}^{T}\right)$

Non-bold characters denote the absolute value of the corresponding vector and hats denote the normalization of a vector. In general, the modes have (realexponential $\times t$ power) time dependence. For modes $k=l$, matrix $\mathbf{P}_{i j k}$ projects $\Delta \mathbf{c}_{0}$ onto the space spanned by $\mathbf{x}_{i j k}$ along the directions of the other generalized right eigenvectors, and this projection will change purely exponentially in time.

\section{Interpretation of the complex modes in the general case}

Complex eigenvalues and eigenvectors always appear in conjugated pairs. Let us denote real and imaginary parts of each quantity by subscripts $R$ and $I$, respectively. Expanding reciprocal relations $\mathbf{y}^{+} \mathbf{x}=1$ and $\mathbf{y}^{+} \overline{\mathbf{x}}=0$, and fulfilling their real and imaginary parts simultaneously, the following orthonormality relations can be derived:

$$
\mathbf{y}_{R}^{T} \mathbf{x}_{I}=\mathbf{y}_{I}^{T} \mathbf{x}_{R}=0 \quad 2 \mathbf{y}_{R}^{T} \mathbf{x}_{R}=2 \mathbf{y}_{I}^{T} \mathbf{x}_{I}=1
$$

A conjugated pair of complex modes describes the superposition of two nonparallel oscillatory modes with the same (real-exponential $\times t$ power) amplitude dependence:

$$
\begin{aligned}
& \frac{t^{k-l}}{(k-l) !}\left(e^{\lambda t} \mathbf{F}_{l \rightarrow k}+\overline{e^{\lambda t} \mathbf{F}_{l \rightarrow k}}\right)=\frac{t^{k-l}}{(k-l) !} e^{\lambda_{R} t}\left[\cos \left(\lambda_{I} t\right) \cdot 2 \mathbf{F}_{l \rightarrow k, R}+\sin \left(\lambda_{I} t\right) \cdot\left(-2 \mathbf{F}_{l \rightarrow k, I}\right)\right], \\
& 2 \mathbf{F}_{l \rightarrow k, R}=2\left(\mathbf{x}_{k R} \mathbf{y}_{l R}^{T}+\mathbf{x}_{k I} \mathbf{y}_{l I}^{T}\right) \quad-2 \mathbf{F}_{l \rightarrow k, I}=2\left(-\mathbf{x}_{k I} \mathbf{y}_{l R}^{T}+\mathbf{x}_{k R} \mathbf{y}_{l I}^{T}\right) .
\end{aligned}
$$


All previous relationships that define generalized eigenvectors remain true if we multiply all right eigenvectors of a Jordan chain with a non-zero complex number (that is $\mathbf{x}_{i j k} \rightarrow \mathbf{x}_{i j k} \cdot z$ ) and simultaneously divide all left eigenvectors with its conjugate $\left(\mathbf{y}_{i j k} \rightarrow \mathbf{y}_{i j k} / \bar{z}\right)$. Using this transformation, it is possible to achieve that one of the right eigenvectors of a Jordan chain of a complex eigenvalue will have real and imaginary parts perpendicular to each other $\left(\mathbf{x}_{k R}^{T} \mathbf{x}_{k I}^{T}=0\right.$ ). This selection doesn't change the motion described by this mode, but makes easier to visualize it. The operations described by matrix $2 \mathbf{F}_{l \rightarrow k, R}$ are skew-projections onto directions $\hat{\mathbf{x}}_{l R}$ and $\hat{\mathbf{x}}_{l I}$, rotations of these components to directions $\hat{\mathbf{x}}_{k R}$ and $\hat{\mathbf{x}}_{k I}$, and rescalings by factors $x_{k R} / x_{l R}$ and $x_{k I} / x_{I I}$, respectively.

$\left[\begin{array}{c}\hat{\mathbf{x}}_{k R}^{T} \\ \hat{\mathbf{x}}_{k I}^{T}\end{array}\right] \cdot 2 \mathbf{F}_{l \rightarrow k, R} \cdot\left[\begin{array}{ll}\hat{\mathbf{x}}_{l R} & \hat{\mathbf{x}}_{l I}\end{array}\right]=\left[\begin{array}{cc}x_{k R} / x_{l R} & 0 \\ 0 & x_{k I} / x_{l I}\end{array}\right]$

The operation described by matrix $-2 \mathbf{F}_{l \rightarrow k, I}$ can be traced back to $2 \mathbf{F}_{l \rightarrow k, R}$ by a subsequent clockwise rotation with an angle of $90^{\circ}$ and an additional rescaling with $x_{k R} / x_{k I}$ and $x_{k I} / x_{k R}$.

$\left[\begin{array}{c}\mathbf{x}_{k R}^{T} \\ \mathbf{x}_{k I}^{T}\end{array}\right] \cdot\left(-2 \mathbf{F}_{l \rightarrow k, I}\right) \cdot\left[\begin{array}{ll}\hat{\mathbf{x}}_{l R} & \hat{\mathbf{x}}_{l I}\end{array}\right]=\left[\begin{array}{cc}x_{k R} / x_{k I} & 0 \\ 0 & x_{k I} / x_{k R}\end{array}\right]\left[\begin{array}{cc}0 & 1 \\ -1 & 0\end{array}\right]\left[\begin{array}{cc}x_{k R} / x_{l R} & 0 \\ 0 & x_{k I} / x_{l I}\end{array}\right]$

Thus, the motion defined in equation (17) can be characterized by a harmonic elliptical motion and the lengths of its principal axes change according to time-dependence of (real-exponential $\times t$ power). For modes $k=l$, the matrix $2 \mathbf{P}_{k R}=2 \mathbf{F}_{k \rightarrow k, R}$ describes a skew-projection onto the space spanned by normalized orthogonal basis vectors $\hat{\mathbf{x}}_{k R}$ and $\hat{\mathbf{x}}_{k I}$. The operation described by matrix $-2 \mathbf{P}_{k I}=-2 \mathbf{F}_{k \rightarrow k, I}$ can be traced back to $2 \mathbf{P}_{k R}$ like in equation (20). This mode describes a harmonic elliptical motion with axes having exponential time-dependence.

\section{SIGNIFICANCE OF THIS APPROACH}

In detailed kinetic models of tropospheric chemical reactions, many chemically similar intermediates are considered and, due to the lack of information, identical rate coefficients (called "generic rate coefficients" [8], 
[9]) are assumed for all similar reactions of these intermediates. The situation is alike for models of low-temperature hydrocarbon combustion. The consequence is that for several such models the Jacobian will be non-diagonalizable and the time-dependence of a perturbation can be evaluated only using the general approach given in this paper. A transient mode discussed above also has a " $t$ power factor" in its time dependence, and therefore it initially has zero amplitude, then reaches a maximum and finally decays to zero. Such a transient mode may have an increased decay time compared to the corresponding mode having purely exponential decay. A purely exponential mode and a transient mode could be contrasted by comparing the times needed for their amplitudes to reach the same small fixed threshold. Maas and Pope [4] have met the numerical problem of finding the eigenvectors in the degenerate case and they applied a Schur decomposition of the Jacobian. This approach solved the problem, but prevented the realization of the modes having non-exponential decay. Taking into account this general evaluation of the matrix exponential in the CSP and ILDM methods would allow an improved determination of the relaxation times of modes. Note that species lumping can be based on the lifetimes of species [10]. Such a lumping results in the elimination of many similar eigenvalues, decreasing the chance of degeneracy.

\section{EXAMPLE SYSTEM}

Assume that chemical transformation $\mathrm{A} \rightarrow \mathrm{B}$ is catalysed by compound $\mathrm{C}$. Also, assume that the concentration of $\mathrm{A}$ is kept constant; product $\mathrm{B}$ and catalyst $\mathrm{C}$ has first order decay according to the following equations:

\begin{tabular}{|c|c|c|}
\hline $\mathrm{A}+\mathrm{C} \rightarrow \mathrm{B}+\mathrm{C}$ & $k_{1}$ & R1 \\
\hline $\mathrm{B} \rightarrow \mathrm{P}$ & $k_{2}$ & R2 \\
\hline $\mathrm{C} \rightarrow \mathrm{Q}$ & $k_{2}$ & R3 \\
\hline
\end{tabular}

Let us examine the perturbation of the concentrations of B and $\mathrm{C}$, and apply perturbations $\Delta b_{0}$ and $\Delta c_{0}$. The production rates, the Jacobian and Jordan matrices are the following:

$\frac{\mathrm{d}}{\mathrm{d} t}\left[\begin{array}{l}b \\ c\end{array}\right]=\left[\begin{array}{c}k_{1} a c-k_{2} b \\ -k_{2} c\end{array}\right] \Rightarrow \mathbf{J}=\left[\begin{array}{cc}-k_{2} & k_{1} a \\ 0 & -k_{2}\end{array}\right] \Rightarrow \boldsymbol{J}=\left[\begin{array}{cc}-k_{2} & 1 \\ 0 & -k_{2}\end{array}\right]$. 


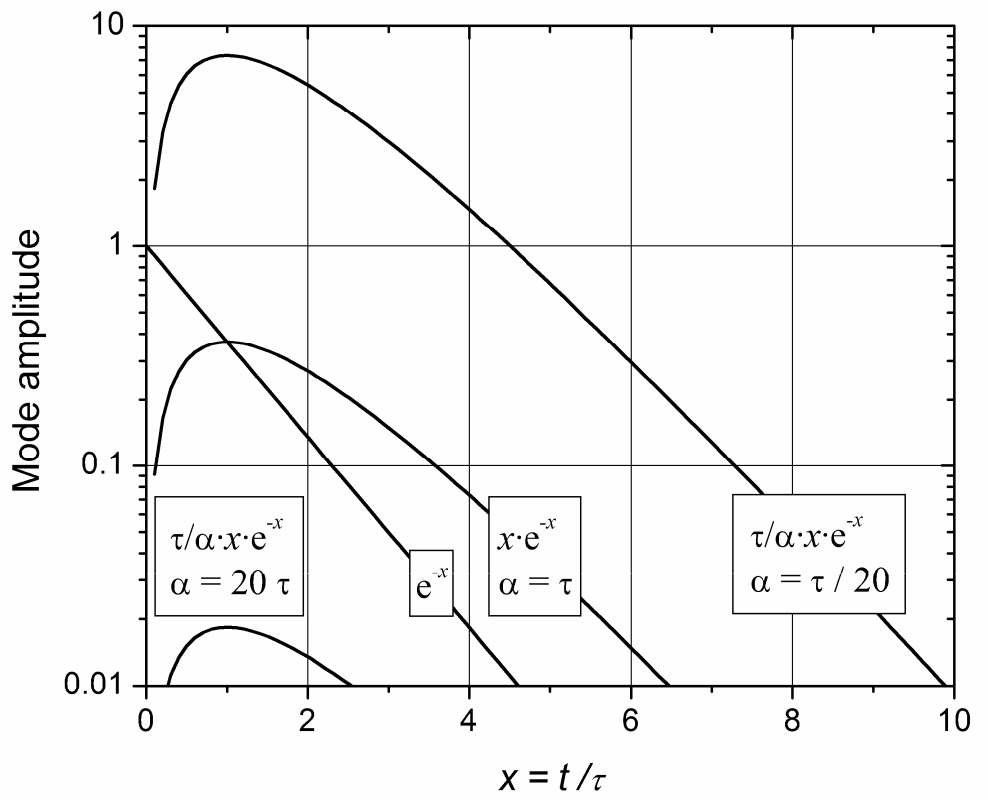

Fig. 1. Time evolution of the modes having purely exponential decay $e^{-t / \tau}$, and also having $(t / \alpha) e^{-t / \tau}$ change for cases $\alpha=20 \tau, \quad \alpha=\tau, \quad \alpha=\tau / 20$ after perturbations $\Delta b_{0}=\Delta c_{0}=1$

In this case, the Jacobian is constant and therefore equation (4) is exact. The Jacobian has a degenerate eigenvalue $\lambda=-k_{2}$, leading to time scale $\tau=-\lambda^{-1}=k_{2}^{-1}$. This is the simplest example for the problem of degenerate Jacobian, discussed in the previous sections. Introducing time constant $\alpha=\left(k_{1} a\right)^{-1}$, the generalized eigenvectors and the evolutions of perturbations are

$\mathbf{P}=\left[\begin{array}{cc}\alpha^{-1} & 0 \\ 0 & 1\end{array}\right], \quad \mathbf{P}^{-1}=\left[\begin{array}{cc}\alpha & 0 \\ 0 & 1\end{array}\right], \quad\left[\begin{array}{l}\Delta b \\ \Delta c\end{array}\right]=e^{-t / \tau}\left[\begin{array}{cc}1 & t / \alpha \\ 0 & 1\end{array}\right]\left[\begin{array}{l}\Delta b_{0} \\ \Delta c_{0}\end{array}\right]$

This means that the decay of $\Delta c$ is purely exponential according to lifetime $\tau$. The change of $\Delta b$ follows function $\Delta b=e^{-t / \tau} \Delta b_{0}+(t / \alpha) e^{-t / \tau} \Delta c_{0}$, which is composed of two modes, thus no similar time scale can be associated to it. Figure 1 shows that the amplitude of the " $t$ power" modes and the time required to reach one hundredth of the initial perturbation can be either significantly smaller or larger than those of the purely exponential one, depending on the 
ratio of time constant $\alpha$ and characteristic time $\tau$. Quantity $\alpha^{-1}$ is identical to scaling factor $x_{i j k} / x_{i j l}$ in equation (15).

\section{CONCLUSION}

This paper demonstrates that the time scales of a system, that is the decay times of modes after a concentration perturbation depend on the properties of the Jacobian. If the Jacobian is diagonalizable, then characteristic time $\tau=1 /\left|\lambda_{R}\right|$ belongs to eigenvalue $\lambda$. If the Jacobian is not diagonalizable, then some of the modes will evolve in time according to function $(t / \alpha)^{n} e^{\lambda t}$, where $\alpha$ is a time constant and $n>0$ is a small integer. In this case, the decay is not exponential and can be prolonged if time constant $\alpha$ is small compared to the characteristic time of the mode.

Acknowledgement. The support of OTKA grant T68256 and the helpful comments of Drs János Tóth and Alison Tomlin are greatly acknowledged.

\section{REFERENCES}

1. A.S. Tomlin, T. Turányi, M.J. Pilling: Low-temperature combustion and autoignition, pp. 293-437, Elsevier, Amsterdam 1997.

2. T. Turányi, A.S. Tomlin, M.J. Pilling: J. Phys. Chem., 97, 163 (1993).

3. S.H. Lam, D.A. Goussis: Proc. Combust. Inst., 22, 931 (1988).

4. U. Maas, S.B. Pope: Combust. Flame, 88, 239 (1992).

5. T. Lu, Y. Ju, C.K. Law: Combust. Flame, 126, 1445 (2001).

6. A.S. Tomlin, L. Whitehouse, R. Lowe, M.J. Pilling: Farad. Discuss., 120, 125 (2001).

7. V. Prasolov: Problems and Theorems in Linear Algebra, Amer. Math. Soc., 1994.

8. S.M. Saunders, M.E. Jenkin, R.G. Derwent, M.J. Pilling: Atmos. Chem. Phys., 3, 161 (2003).

9. M.E. Jenkin, S.M. Saunders, V. Wagner, M.J. Pilling: Atmos. Chem. Phys., 3, 181 (2003).

10. L.E. Whitehouse, A.S. Tomlin, M.J. Pilling: Atmos. Chem. Phys., 4, 2057 (2004). 